\title{
File Transfer Protocol
}

National Cancer Institute

\section{Source}

National Cancer Institute. File Transfer Protocol. NCI Thesaurus. Code C100047.

A network protocol designed to move files between a computer and a host over a TCP network, such as the Internet. 